\title{
INVESTIGATIONS ON THE MANUFACTURING AND MECHANICAL PROPERTIES OF SPUN YARNS MADE FROM STAPLE CF FOR THERMOSET COMPOSITES
}

\author{
Mir Mohammad Badrul Hasan*, Martin Hengstermann, Rebekka Dilo, Anwar Abdkader and Chokri Cherif \\ Institute of Textile Machinery and High Performance Material Technology (ITM) \\ Technische Universität Dresden, Germany \\ ${ }^{*}$ Corresponding author: Mir_Mohammad_Badrul.Hasan@tu-dresden.de
}

\begin{abstract}
:
This article reports the results of investigations carried out to produce yarns consisting of staple carbon fiber (CF) obtained from process waste for the manufacturing of composites suitable especially for thermoset applications. For this purpose, a comparative analysis is done on processability between 100\% staple CF and 60 weight $\%$ staple CF mixed with 40 weight\% PVA fibers in carding, drawing and spinning process. The hybrid yarns are produced by varying twist level. The PVA fibers of the hybrid yarn are then dissolved using hot water treatment. The mechanical properties of yarns consisting of 100\% staple CF and hybrid yarns consisting of staple CF and PVA before and after hot water treatment are investigated. Furthermore, test specimen is also prepared by impregnating $100 \%$ staple CF yarn and the hybrid yarns (after the dissolving of PVA) with epoxy resin. The results of the tensile test of the yarns in consolidated state reveals that the hybrid yarn produced with $80 \mathrm{~T} / \mathrm{m}$ after hot water treatment exhibits approximately $75 \%$ of the tensile strength of virgin filament tow, and it is expected that the hybrid yarns can be applied for the manufacturing of thermoset based composites for load bearing structures.
\end{abstract}

\section{Keywords:}

Poly vinyl alcohol fiber, carbon fiber, hybrid yarn, carding, spinning

\section{Introduction}

Because of their excellent strength, rigidity and damping properties, as well as low weight and high resistances to impact and corrosion, the demand and usage of carbon fiber reinforced composites (CFRC) is growing steadily for example in aerospace, automobiles and rotor blades [1].The CFRCs are comprised of CF reinforcement combined with a binding matrix, and they are classified mainly into thermoset and thermoplastic composites depending on the type of polymer matrix. Because of the ease of manufacturing, higher thermal stability, excellent fatigue strength and good fiber to matrix adhesion, CFRCs are preferentially manufactured based on a thermoset matrix (more than $75 \%$ of all the composites) [2, 3].

On the other hand, with an increased demand and usage of CFRCs, effective methods to re-use waste CF materials, which are recoverable either from the process scraps or from the end-of-life components, are attracting increased attention. A major portion of such waste CF is available in the form of staple fibers ranging from 1 to $20 \mathrm{~cm}$ [4]. These circumstances have led to a focused attention on the need to find effective methods to re-use waste CF materials (recovered from process scraps) and recycled CF (rCF) (recovered from the end-of-life components). The ability to manufacture yarns (from short CF) would greatly expand the application of CFRC in vehicles and other structures, where cost reduction is extremely important.

However, little information can be found so far, regarding the spinning of yarns consisting of $100 \%$ staple CF from the literature review. Due to the brittleness, sensitivity to shear stress and lack of natural crimp in CF, the processing of staple $\mathrm{CF}$ into slivers, as well as yarns, is a challenging task compared to the conventional textile fibers.

The manufacturing method to produce spun yarn consisting of $100 \% \mathrm{CF}$, which is widely practiced due to the ease of manufacturing, is the stretch breaking technique in which continuous filaments are converted into short fibers [5]. The most prominent manufacturers of such yarns are Courtaulds' Heltra Division [6], Schappe (Saint-Rembert-en-Bugey, France) [7] and SGL [8]. However, this method is not suitable to align the un-oriented short fibers supplied as the feeding material for the manufacturing of staple yarns. Therefore, a different approach is required for the production of spun yarn consisting of $100 \%$ staple CF.

The manufacturing of hybrid yarns consisting of staple $\mathrm{CF}$ and thermoplastic staple fibers has been reported in [9-13] using ring spinning and in [14] using wrap spinning method. The mixing between staple CF and thermoplastic staple fibers is carried out by carding prior to spinning. Such hybrid yarns are especially suitable for the manufacturing of thermoplastic composites. As the viscosity of thermoplastic melts are very high (e.g., the viscosity of polypropylene is $10^{3}$ Pa.s at $200-270{ }^{\circ} \mathrm{C}$ [15]), the mixing between CF and thermoplastic fibers helps to reduce the flow path of the matrix during the consolidation of composites. As a result, the problems associated with the impregnation of CF with thermoplastic matrix can be avoided. The CF and the 
thermoplastic component work as the reinforcement and the matrix in the composite, respectively.

On the other hand, the yarns required for the manufacturing of thermoset matrix should ideally consist only of reinforcement fibers. As the viscosity of thermoset matrix is relatively low (e.g., the viscosity of cold set polyester resin is $100 \mathrm{mPa}$.s [15]) as compared to that of thermoplastic melts, the pure reinforcement component can easily be impregnated with thermoset matrix. However, the processing only with staple CF without the inclusion of auxiliary fibers (to improve the processability of $\mathrm{CF}$ ) in different process steps for the manufacturing of yarn consisting of $100 \%$ CF is difficult. The auxiliary fibers help to increase the fiber to fiber cohesion and ensure a gentle processing of CF. As a result, a better quality of the yarn can be achieved. Therefore, efforts should be made to reduce the amount of auxiliary fibers. The aim on one hand is to improve the processability of CF and on the other hand to reduce the risk of the deterioration of the mechanical properties of composites as a result of the inclusion of auxiliary fibers.

In this paper, the results of the investigations regarding the manufacturing and mechanical properties of yarns consisting of staple CF suitable for thermoset composites have been reported. For this purpose, two variants of yarns are produced such as:

- Variant 1: yarns purely with $100 \%$ staple CF and

- Variant 2: hybrid yarns consisting of staple CF and polyvinyl alcohol (PVA) staple fibers.

Hybrid yarns are manufactured by mixing staple CF with PVA fibers in order to improve the processability of CF. PVA staple fibers in the form of tufts are mixed with staple CF during carding. The PVA component of the hybrid yarns are then dissolved to obtain yarns consisting of $100 \%$ CF. The aim is to produce CF spun yarns that can be used especially for the manufacturing of thermoset composites, which contain no additional material. The manufacturing of both variants of yarns are carried out using carding, drawing and roving frame machine. The aim of this paper is to explore the effect of using PVA fibers on the processability of the CF and mechanical properties of yarns before and after the dissolving of PVA component.

\section{Experimental details}

\section{$\underline{\text { 2.1 Fiber materials }}$}

For the investigation reported in this study, virgin continuous carbon filament tows SIGRAFIL C50 T050 EPY (SGL) are cut into $60 \mathrm{~mm}$ staple length (with a tolerance of $\pm 2 \mathrm{~mm}$ ) and used for the manufacturing of yarns/hybrid yarns. These virgin staple CFs will work as reinforcement components in the composite and can be regarded as a synonym to the process waste obtained from rest of the spool or from fabric selvedges.

The PVA fibers KURALON K-II (Kuraray Co. LTD. Japan) of $51 \mathrm{~mm}$ staple length are used for the manufacturing of hybrid yarns. The nominal dissolving temperature of the used PVA fibers is $40^{\circ} \mathrm{C}$ in water [16]. In order to obtain yarns consisting of $100 \% \mathrm{CF}$, the PVA component of hybrid yarns is dissolved later on using hot water. The tensile properties of virgin staple CF and PVA fibers are detailed in Table 1.

\subsection{Manufacturing of yarns/ hybrid yarns}

For the manufacturing of hybrid yarns consisting of virgin staple CF with PVA staple fibers, as well as yarns purely with virgin staple CF, first of all, carding is done using a modified laboratory long staple carding machine (Anton Gulliot, Germany) optimized for the processing of staple CF at the Institute of Textile Machinery and High Performance Material Technology (ITM). To ensure a minimum level of fiber damage and smooth running of the carding process, an optimized clearance between different rollers is used (cf. Table 2). The parameters of carding are selected from initial investigations to ensure gentle processing of CF. It is to be mentioned here that several investigations are also carried out by mixing

Table 1: Tensile properties of virgin staple CF and PVA single fiber

\begin{tabular}{|c|c|c|c|}
\hline Characteristics & Virgin staple CF* & PVA [16] & SIGRAFIL filament tow [17] \\
\hline Linear density (dtex) & $0.67 \pm 0.06$ & 2.20 & 33000.00 \\
\hline Tensile strength (GPa) & $3.60 \pm 0.63$ & 0.95 & 4.00 \\
\hline Elongation at break (\%) & $1.64 \pm 0.27$ & 15.00 & 1.70 \\
\hline
\end{tabular}

*Measured according to DIN EN ISO 5079

Table 2: Optimum clearance between different rollers in carding machine

\begin{tabular}{|c|c|}
\hline Rollers & Clearance (mm) \\
\hline Worker-Cylinder & 0.4 \\
\hline Stripper-Cylinder & 0.6 \\
\hline Taker in-Cylinder & 0.5 \\
\hline Cylinder-Doffer & 0.8 \\
\hline
\end{tabular}


different weight \% of PVA with staple CF. However, a better process stability and a gentle processing, as well a better sliver and hybrid yarn quality can be achieved by mixing 40 weight $\%$ of PVA with staple CF. The details about the card wire specification can be found in [9]. The fineness of card webs that are produced is around $26 \mathrm{ktex}$. A $24 \mathrm{~g}$ fiber material (i.e., either $100 \%$ CF or CF mixed with PVA in the required ratio) is fed for the production of each card web. In order to obtain a better mixing between CF and PVA, the fibers are mixed by compressed air of 2 bars for $10 \mathrm{~s}$ in a container prior to carding. The dimension of the produced card webs is kept $150 \times 650 \mathrm{~mm}$ and the width of the card web is adjusted to the maximum width of the drawing zone in order to ensure a smooth feeding into the draw frame. The length of each carded web corresponds $0.7 \mathrm{~m}$. It is to be mentioned here that card webs are used as the input for drawing and no doubling is done for the production of draw frame slivers.

Afterwards, drafting is carried out on the card webs using a modified high performance draw frame RSB-D40 (Rieter, Ingolstadt, Germany) optimized for the gentle processing of staple CF. The modifications include encapsulation of electronic components to avoid any short circuit during the processing of the card web into draw frame slivers. Furthermore, the grooved bottom rollers are replaced with new bottom rollers with a smooth surface. The total draft of 6.46 is kept constant for all the variations in order to produce draw frame slivers of a fineness of around 4 ktex.

Finally, the yarns of fineness around 800 tex are spun using the draw frame slivers on a modified Roving frame $F 15$ (Rieter, Winterthur, Switzerland). A slower rotation speed of the flyers (800 rpm), and decreased feeding speed $(8 \mathrm{~m} / \mathrm{min})$ are selected in order to keep the damage of CF minimal. The gauge and draft for different fiber lengths used in the draw frame and roving frame can be found in Table 3. An overview of card webs, slivers and the yarns/ hybrid yarns that are produced for the investigations is given in Table 4.

\subsection{Fiber to fiber cohesion in draw frame sliver}

The cohesion between the fibers in a fibrous assembly plays an important role in determining the material behavior during spinning process. Without sufficient force of cohesion, it is not possible to pull the slivers, for example, from cans or to draft them on a roving frame without drafting error or interruptions. For this purpose, a Rothschild cohesion meter R-2020 is used to obtain an idea about drafting behavior of slivers in the subsequent spinning process. First of all qualitatively, the tests indicate the suitability of slivers for further processing and secondly, quantify the exact force of cohesion. The measurements are done at a speed of $5 \mathrm{~m} / \mathrm{min}$ for $1 \mathrm{~min}$ with a draft of 1.5 using a gauge of $78 \mathrm{~mm}$.

\subsection{Dissolving of PVA}

In order to dissolve the PVA part in the hybrid yarn, the hybrid yarns are treated in a hot water bath at $50{ }^{\circ} \mathrm{C}$ for $30 \mathrm{~min}$. The temperature and time of hot water treatment are selected from initial investigations to obtain the optimum result. The amount of PVA that is dissolved is determined by the measurement of yarn fineness, as well as by thermo-gravimetric analysis (TGA). TGA is carried out on the hybrid yarns (i.e., CF mixed with PVA), as well as on yarn consisting of $100 \% \mathrm{CF}$ to have an understanding on the extent of PVA remaining on the hybrid yarns. In order to carry out TGA, the TA Instruments Q500 within the temperature range $30-600^{\circ} \mathrm{C}$ in air with a flow rate of $50 \mathrm{~mL} / \mathrm{min}$ and a heating rate of $40^{\circ} \mathrm{C} / \mathrm{min}$ is used. The temperature range of $30-600{ }^{\circ} \mathrm{C}$ is selected, as the reduction of mass of $\mathrm{CF}$ as a result of oxidation is low in this temperature range [18]. Furthermore, hybrid yarns are analyzed using scanning electron microscopic (SEM) image after hot water treatment.

\subsection{Preparation of consolidated test specimen with yarns}

In order to obtain an idea about the tensile strength of the yarns (i.e., yarn produced from $100 \%$ staple CF, as well as the hybrid

Table 3: Gauge and draft of break and main draft zone used in draw frame and roving frame

\begin{tabular}{|c|c|c|c|c|}
\hline \multirow{2}{*}{ Drafting zone } & \multicolumn{2}{|c|}{ Draw frame } & \multicolumn{2}{c|}{ Roving frame } \\
\cline { 2 - 5 } & Gauge(mm) & Draft & 71 & 1.05 \\
\hline Break draft & 65 & 1.15 & 74 & 4.78 \\
\hline Main draft & 65 & 5.62 & & Draft \\
\hline
\end{tabular}

Table 4. An overview of card webs and the yarns/ hybrid yarns produced for the investigations

\begin{tabular}{|c|c|c|c|c|c|c|}
\hline \multicolumn{3}{|c|}{ Card web } & $\begin{array}{l}\text { Draw frame } \\
\text { sliver }\end{array}$ & \multicolumn{3}{|c|}{ Yarns/ hybrid yarns } \\
\hline $\begin{array}{c}\text { Weight } \% \text { of } \\
\text { CF }\end{array}$ & $\begin{array}{c}\text { Weight } \% \text { of } \\
\text { PVA }\end{array}$ & $\begin{array}{l}\text { Fineness } \\
\text { (ktex) }\end{array}$ & $\begin{array}{l}\text { Fineness } \\
\text { (ktex) }\end{array}$ & Twist (T/m) & $\begin{array}{l}\text { fineness } \\
\text { (tex) }\end{array}$ & Identification \\
\hline 100 & - & \multirow{3}{*}{26} & \multirow{3}{*}{4} & 80 & \multirow{3}{*}{800} & 100\%CF_0\%PVA_80T \\
\hline \multirow{2}{*}{60} & \multirow{2}{*}{40} & & & 80 & & $60 \%$ CF_40\%PVA_80T \\
\hline & & & & 118 & & $60 \%$ CF $40 \%$ PVA $118 \mathrm{~T}$ \\
\hline
\end{tabular}


yarns after dissolving of the PVA component) in consolidated state with matrix, the single yarn is impregnated with thermoset resin. For this purpose, the yarns are impregnated in stretched condition with thermoset resin Biresin CR-81 (Sika Deutschland $\mathrm{GmbH}$ ) and kept at room temperature for 24 hours for consolidation. Then the specimen of $250 \mathrm{~mm}$ length is cut and the test specimen is prepared by fixing cap strips on both sides of the consolidated yarn specimen. Furthermore, in the same way a reference test specimen is prepared from the continuous carbon filament tow SIGRAFIL C50 T050 EPY (SGL).

\subsection{Test of mechanical properties of hybrid yarns}

Tensile tests of the manufactured yarns/hybrid yarns are carried out according to ISO 3341. For this purpose, a tensile strength testing device Zwick type Z 2.5 (Zwick $\mathrm{GmbH}$ and Co., Germany) with special return clamps and external strain measuring is used. The tensile testing of the hybrid yarns are performed before and after hot water treatment to find out the effect of the dissolving of PVA on yarn strength. Samples of $250 \mathrm{~mm}$ yarn length are used. The test speed is set to 100 $\mathrm{mm} / \mathrm{min}$ and the initial load is kept at $0.5 \mathrm{cN} /$ tex. The tensile force versus deformation is recorded and 10 measurements are taken to calculate the average value for each type of yarn. The stress-strain behavior is evaluated using the testXpert ${ }^{\mathrm{B}}$ software. The instrument is located in a temperature and relative humidity controlled laboratory maintained at $20 \pm 2^{\circ} \mathrm{C}$ and $65 \pm 2 \%$, respectively. In order to compare different yarns, the tenacity is calculated using Equation 1:

$$
\text { Tencity }(c N / \text { Tex })=\frac{F_{\max }(N) \times 100}{\text { Yarn fineness }(\text { Tex })}
$$

Where, Fmax is the maximum breaking force for yarn.

\subsection{Tensile testing of consolidated test specimen}

The testing of the tensile properties of the consolidated test specimen with different yarns is performed on the testing device Zwick type Z 100 (Zwick GmbH and Co., Germany) in accordance with the Standard DIN EN ISO 527-5. A minimum of 10 measurements are taken to obtain the average value. The cross head speed of $2 \mathrm{~mm} / \mathrm{min}$ is used for the tensile test and the elongation is measured using an optical sensor. The strength of the consolidated test specimen is calculated using Equation 2:

$$
\text { Strength }(M P a)=\frac{F_{\max }(N)}{A_{0}}
$$

Where $A_{0}$ is the cross-sectional area in $\mathrm{mm}^{2}$ before straining, which can be calculated using Equation 3:

$$
A_{0}\left(\mathrm{~mm}^{2}\right)=\frac{\text { Fineness }(\text { Tex })}{\rho \times 10^{3}}
$$

Where $\rho$ is the density of yarns in $\mathrm{g} / \mathrm{cc}$. In the case of yarns with $100 \% \mathrm{CF}$ and filament tow, $\rho$ is $1.79 \mathrm{~g} / \mathrm{cc}$. For hybrid yarns after hot water treatment, $\rho$ is calculated as 1.74 and $1.66 \mathrm{~g} / \mathrm{cc}$ for 80 and $118 \mathrm{~T} / \mathrm{m}$ respectively, depending on the amount of PVA in the hybrid yarn according to Equation 4 as follows:

$$
\rho=\frac{m}{v}
$$

Where $m$ is the weight of yarn in $g$ and $v$ is volume of yarn in $\mathrm{cm}^{3} . m=\frac{T_{t} \cdot l}{10^{5}}$ is calculated according to the definition of tex. Where $T_{t}$ is the fineness of yarn in Tex and $l$ is the length of 1 $\mathrm{cm}$ yarn. Putting $v=A \cdot l$ (is the area of yarn in $\mathrm{cm}^{2}$ ) and the value of $m$ in Equation 4, Equation 5 can be derived:

$$
\rho=\frac{T_{t}}{10^{5} \cdot A}
$$

$A$ is the sum of area of CF and PVA component in yarn. The area of individual component (i.e., CF or PVA in yarn) is calculated according to Equation 6:

$$
A_{C F / P V A}=\frac{T_{t C F / P V A}}{10^{5} \cdot \rho_{C F / P V A}}
$$

In order to calculate the density of hybrid yarns, the value of $1.36 \mathrm{~g} / \mathrm{cc}$ is used for the density of PVA [19].

\section{Results and Discussion}

\subsection{Influence of PVA on the processability of CF in different process steps}

During the investigations, it can be seen that the mixing of PVA with staple CF influences carding, drawing and spinning process significantly. As the fiber to fiber cohesion in the case of $100 \%$ CF is low because of the smooth surface of CF, a higher loss of fiber material (calculated by measuring the weight of the fiber material before and after carding process) can be observed during carding (cf. Figure 1 (a)). In the case of mixing staple CF with PVA, the increase of fiber to fiber cohesion expedites the carding process and a gentle processing of $\mathrm{CF}$ can be achieved. This in turn influences the sliver compactness and process stability for the further processing of slivers on the roving frame. In Figure 2, the slivers manufactured from 100\% CF and by mixing PVA with CF can be seen.

The results of fiber to fiber cohesion in draw frame slivers show that the draw frame slivers produced from $100 \%$ CF possesses significantly lower cohesion force compared to that of sliver produced by mixing CF with PVA fibers (cf. Figure 1 (b)). It is also to be noticed that the deviations of the measured values in the case of $100 \% \mathrm{CF}$ is also relatively higher compared to that with 60 weight $\%$ CF and 40 weight\% PVA. This also indicates the inconsistency of the band quality, which influences the yarn quality subsequently. From the visual evaluation, it can be seen that the irregularity in yarn produced with $100 \%$ CF is higher compared to that in hybrid yarns (cf. Figure 3). The inclusion of PVA improves the processability of slivers on draw frame, as well as on the roving frame. As a result, better yarn quality can be achieved by mixing PVA with CF staple fibers.

\subsection{Characterizations of the amount of PVA}

The hybrid yarns are manufactured with the same fineness of 800 tex by varying the yarn twist. Since, CF content of the manufactured hybrid yarns is 60 weight $\%$, the yarn fineness should be ideally 480 tex in the case of dissolving of PVA component completely. However, from Figure 4, a significant difference in yarn fineness after hot water treatment can be 


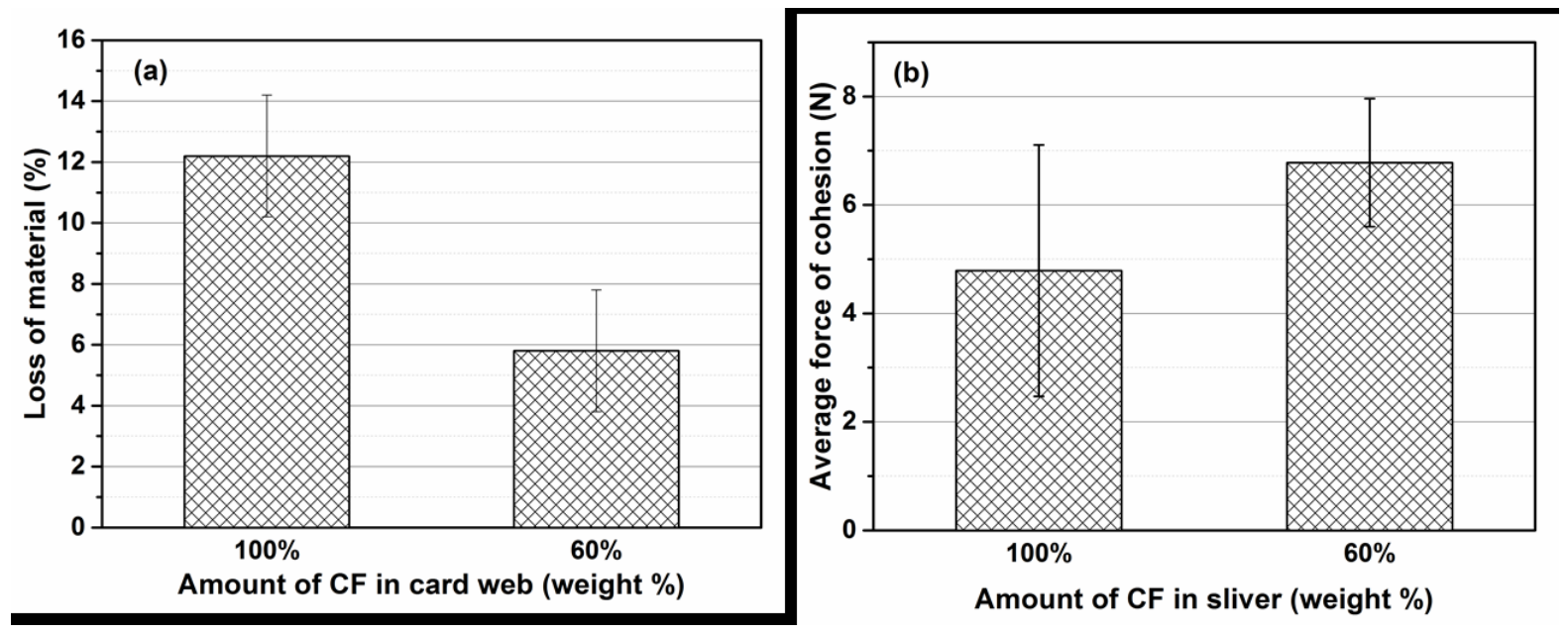

Figure 1: (a) Loss of material during carding; (b) average force of cohesion measured on draw frame slivers

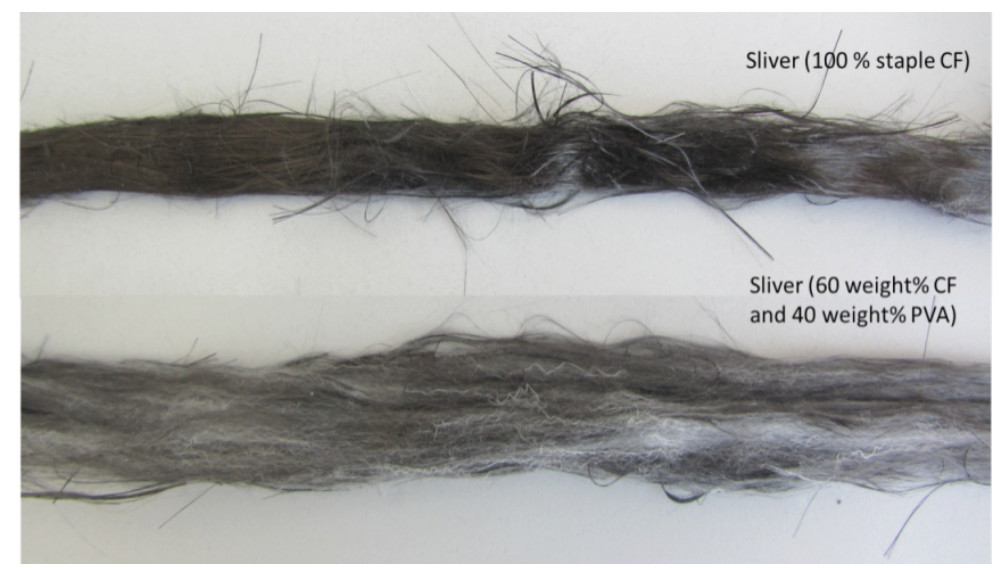

Figure 2: Draw frame slivers manufactured from $100 \%$ staple CF (top) and by mixing 60 weight $\%$ staple with 40 weight $\%$ PVA (bottom)

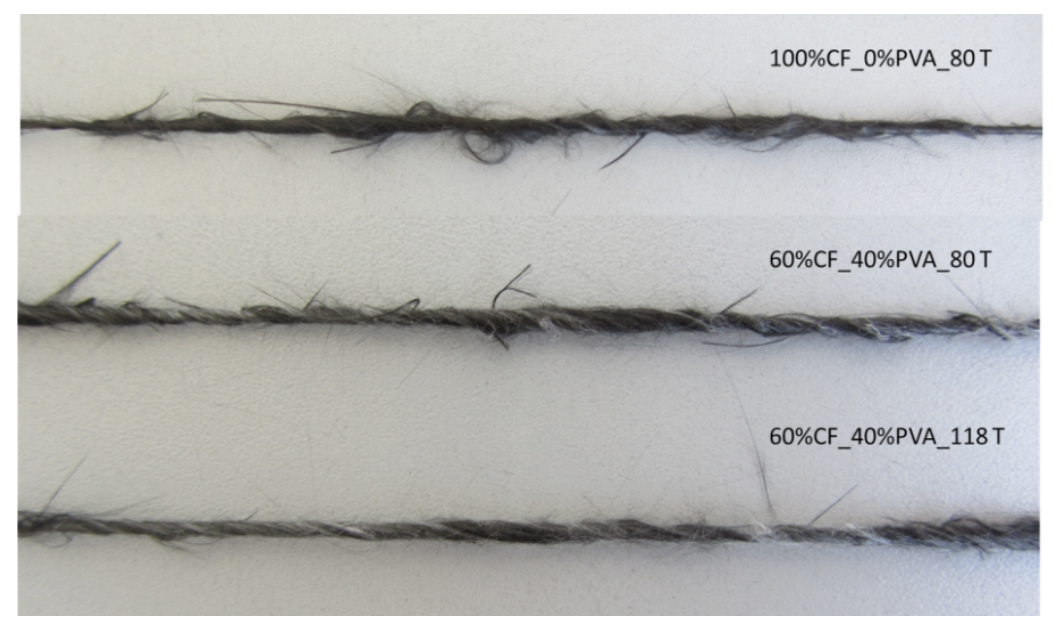

Figure 3: Appearance of yarns/ hybrid yarns produced for the investigations

observed due to the variation of yarn twist. The fineness of the hybrid yarns is found to be 540 and 652 tex in the case of 80 and $118 \mathrm{~T} / \mathrm{m}$ respectively, after treating the hybrid yarns with hot water. Therefore, the calculated amount of PVA (determined by yarn fineness measurement) remaining on the hybrid yarn is 11 weight $\%$ and 26 weight $\%$ in the case of 80 and $118 \mathrm{~T} / \mathrm{m}$ respectively. The higher yarn compactness as a result of higher twisting causes difficulty in dissolving the PVA fibers.
The result of TGA also verifies the effect of twist on the amount of PVA remaining on the hybrid yarn as shown in Figure 5. It can been observed from the TGA curves that the loss of weight in hybrid yarns (consisting of 60 weight\% staple CF and 40 weight $\%$ PVA) is significantly higher as compared to that of the yarn with $100 \% \mathrm{CF}$ above $300^{\circ} \mathrm{C}$ as a result of oxidation of the PVA component. The loss of weight of the hybrid yarns after the hot water treatment indicates that the hybrid yarns are not 
completely free of PVA. In Figure 6, the PVA remaining after the hot water treatment on CF can be seen. The rest of dissolved PVA seems to make a layer on the hybrid yarn.

\subsection{Influence of twist and PVA on the mechanical properties of hybrid yarns}

The results of the tensile strength measurement of the yarns/ hybrid yarns are illustrated graphically in Figure 7. By comparing the yarn consisting of $100 \%$ staple CF with hybrid yarns before hot water treatment, it can be seen that the yarn with $100 \%$ staple CF possesses the highest tenacity. This is because of the difference of CF amount in yarn. As the PVA does not contribute to the strength of yarn significantly, therefore, the strength of hybrid yarns (in which CF content is 60 weight\%) is lower as compared to that of the yarn with $100 \%$ staple CF.

The tenacity of hybrid yarn is slightly lower in the case of $118 \mathrm{~T} / \mathrm{m}$ compared to of the yarn with $80 \mathrm{~T} / \mathrm{m}$. This could be attributed to higher twisting angle of CF to the direction of load bearing axis (i.e., yarn axis) and/or higher damage due to higher twisting.

It is interesting to find that the strength of hybrid yarns for both levels of twisting increases significantly after hot water treatment. From the TGA investigations, as well as the SEM images, it is found that the hybrid yarns are not completely free of PVA. The PVA remaining on the hybrid yarns act as a coating layer on CF and increases the fiber to fiber adhesion to a great extent. As a result, the tenacity and E-module of hybrid yarn increases after hot water treatment, as can be seen from the stress strain curves of the yarns illustrated in Figure 8. With the increase of E-module, the elongation at break of the hybrid yarns becomes lower.

Furthermore, the difference in yarn strength due to yarn twist becomes higher after the dissolving of PVA as a result of hot water treatment. This can be attributed to the change in the amount of PVA remaining on the hybrid yarn after hot water treatment. The amount of PVA remaining on hybrid
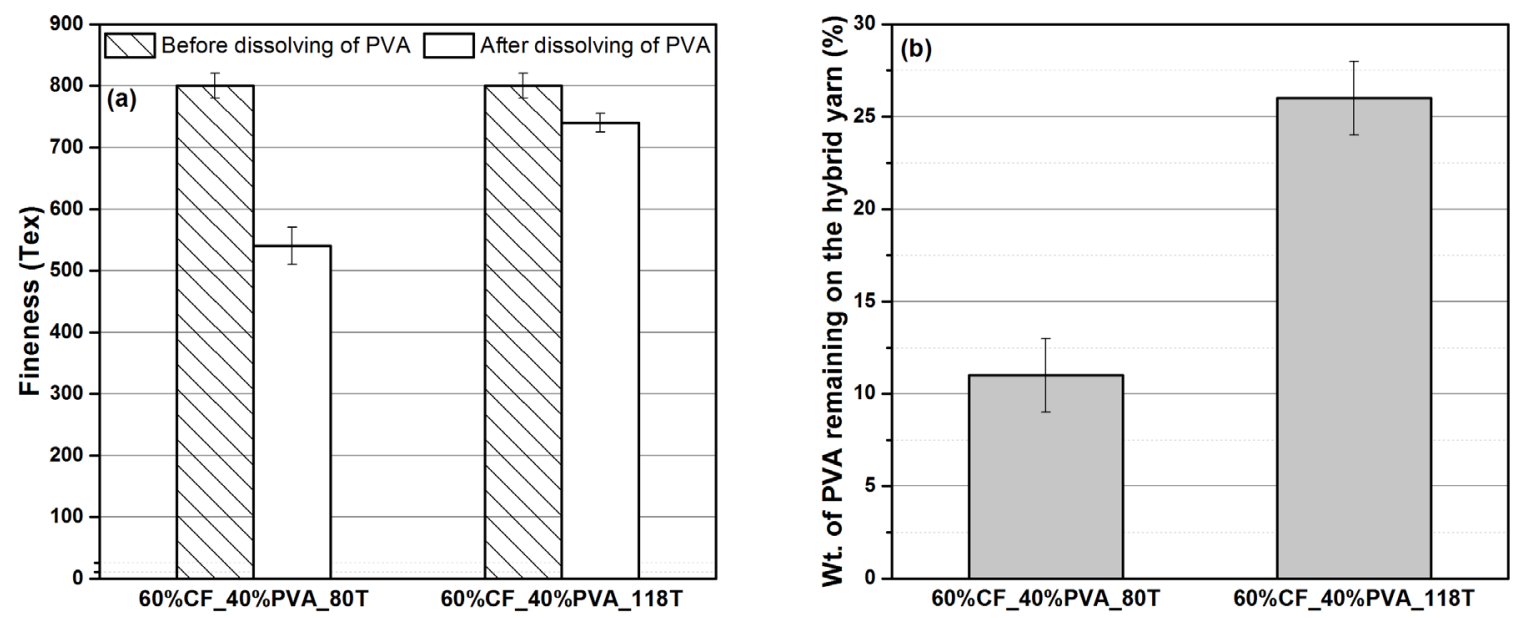

Figure 4: (a) Hybrid yarn fineness before and after dissolving of PVA and (b) the remaining amount of PVA on the hybrid yarn after dissolving of PVA depending on yarn twist

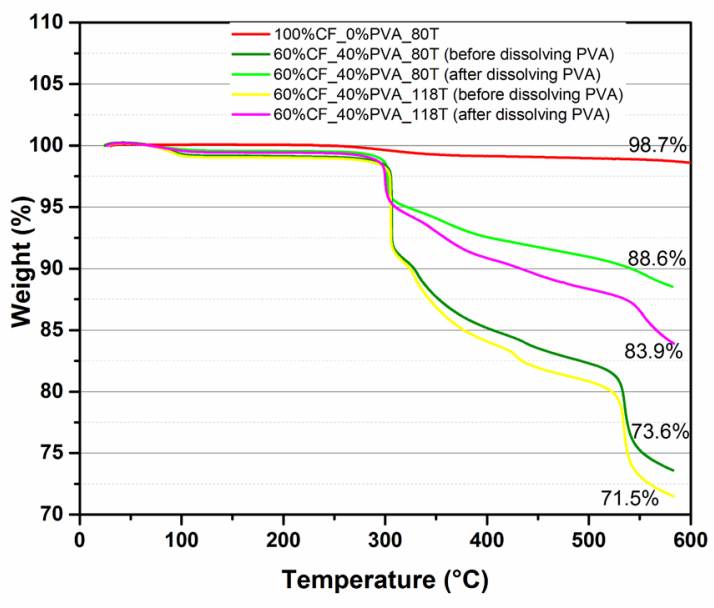

Figure 5: Curves of TGA carried out on yarn /hybrid yarns

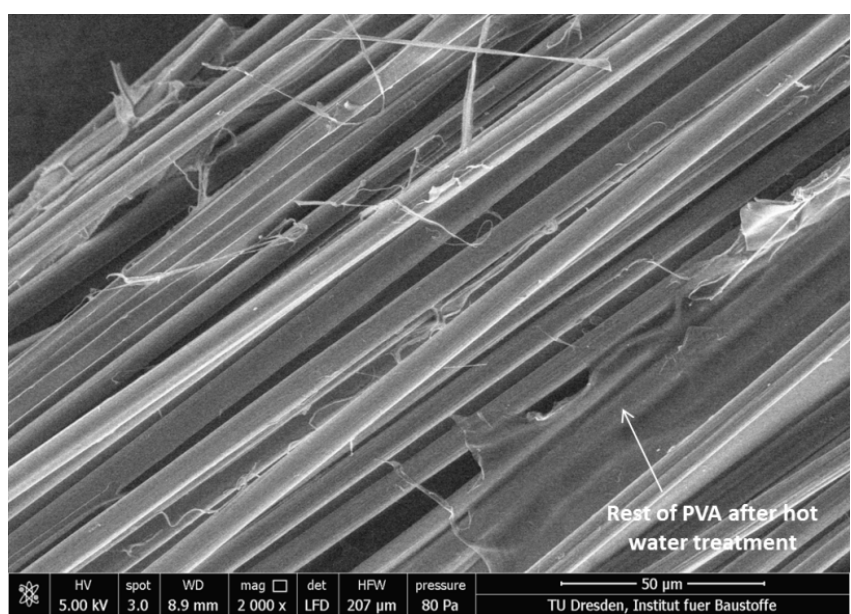

Figure 6: SEM image of $60 \%$ CF_40\%PVA_80T after dissolving of PVA 


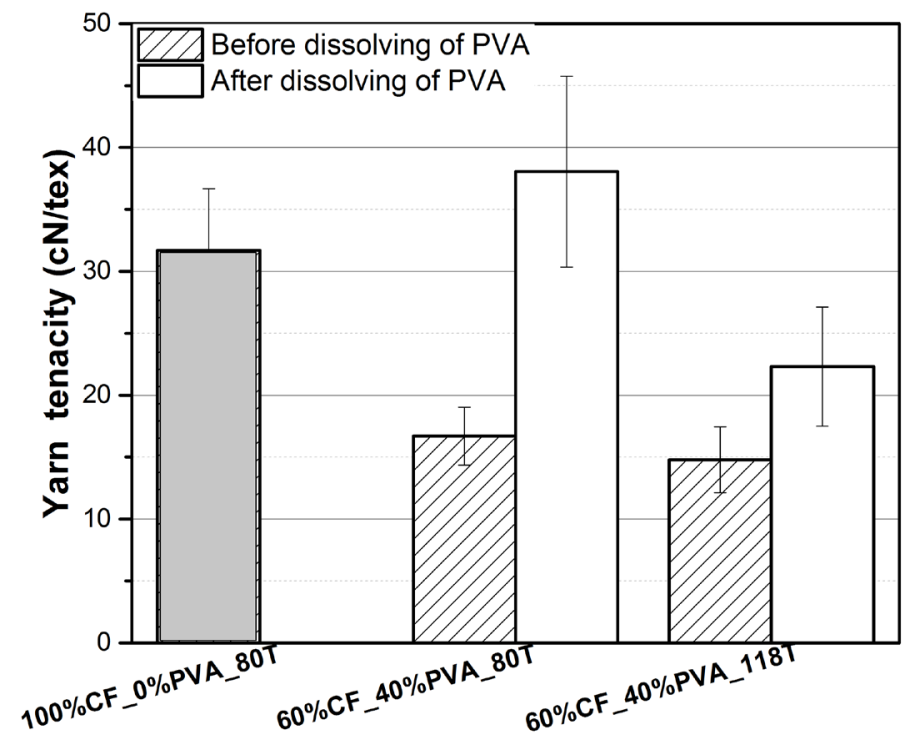

Figure 7: Comparison of hybrid yarn tenacity before and after dissolving of PVA with yarn with $100 \%$ staple CF
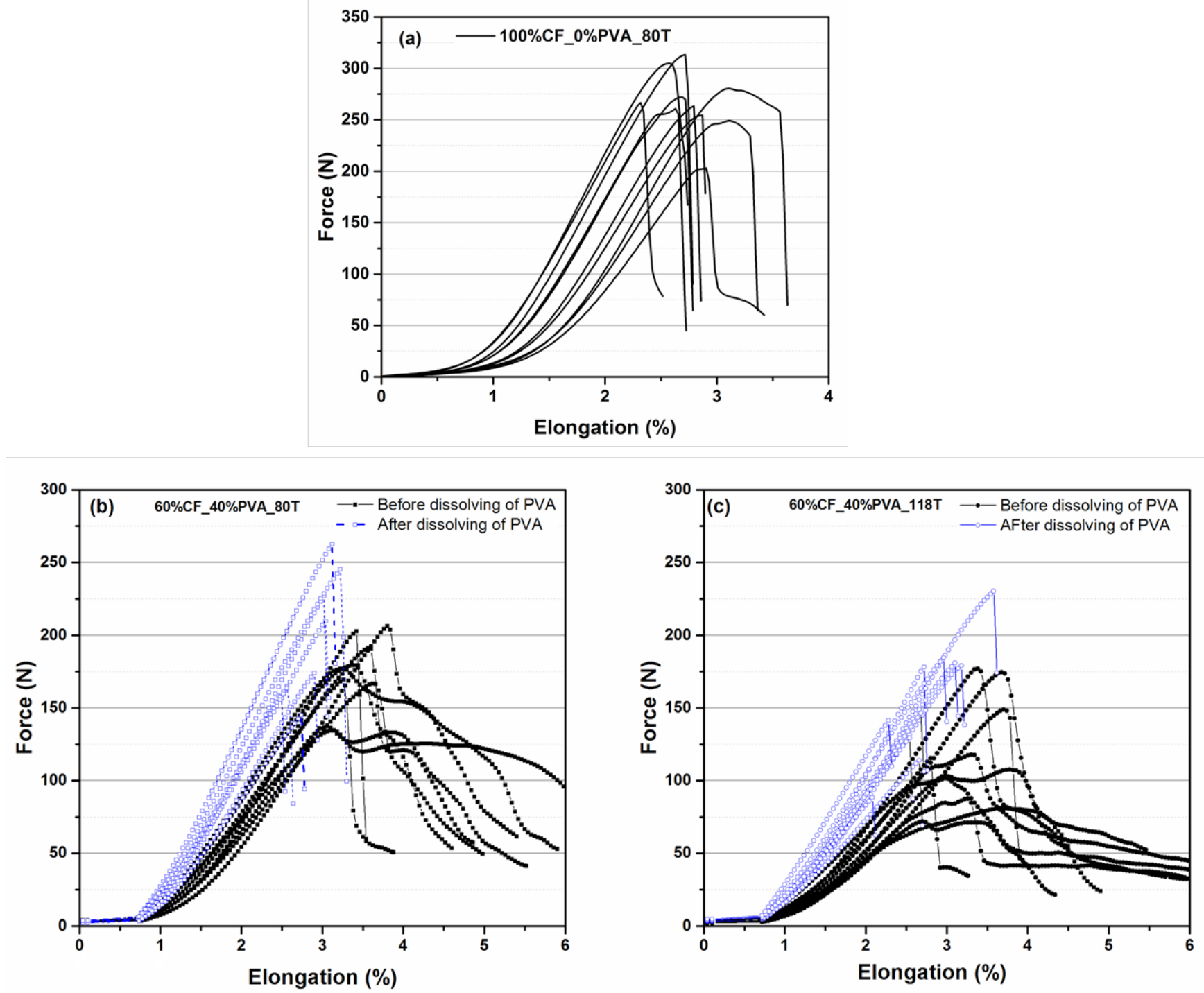

Figure 8: Stress strain curves of yarn consisting of $100 \%$ CF (a) and hybrid yarns made by mixing CF with PVA manufactured with 80 (b) and $118 \mathrm{~T} / \mathrm{m}$ (c) 
yarn manufactured with $118 \mathrm{~T} / \mathrm{m}$ is comparatively higher than that in the hybrid yarn with $80 \mathrm{~T} / \mathrm{m}$ (cf. Figure 4(b) and Table 5). Since, higher the proportion of reinforcing component in the hybrid yarn, higher is the strength of yarn, the change in the tenacity of hybrid yarns becomes higher after hot water treatment. The results of the tensile properties carried out on yarns/hybrid yarns are presented in detail in Table 5.

\subsection{Mechanical properties of hybrid yarns in consolidated state}

In Figure 9, the comparison of tensile strength of consolidated yarns impregnated with epoxy resin is illustrated. Furthermore, the tensile strength of the yarn with $100 \%$ staple CF and hybrid yarns consisting of 60 weight $\%$ CF and 40 weight $\%$
PVA is compared with that of virgin filament tow to get an understanding of the level of strength that can be achieved by spun yarns.

From the comparison of tensile strengths of different yarns manufactured for the investigations, the same tendency was found in the results of tensile tests of hybrid yarn (i.e., before consolidation) after the hot water treatment. The average strength of the test specimen made from $100 \%$ virgin staple CF and filament tow was found to be 1942 and 3222 $\mathrm{MPa}$, respectively. On the other hand, the tensile strength of consolidated hybrid yarn (manufactured with $80 \mathrm{~T} / \mathrm{m}$ ) is approximately $2420 \mathrm{MPa}$, which is around $75 \%$ of the tensile strength of virgin filament yarn. The hybrid yarn manufactured with $80 \mathrm{~T} / \mathrm{m}$ exhibits similar E-module value compared to that of virgin filament tow. Typical stress strain curves of yarns in

Table 5: Tensile properties of the yarns/ hybrid yarns investigated

\begin{tabular}{|l|c|c|c|c|c|}
\hline Yarn specification & $\begin{array}{c}\text { Yarn fineness } \\
\text { (Tex) }\end{array}$ & $\begin{array}{c}\text { Wt.\% of CF } \\
\text { in yarn }\end{array}$ & $\begin{array}{c}\text { Wt.\% of PVA } \\
\text { in yarn }\end{array}$ & $\begin{array}{c}\text { Average tenacity } \\
\text { (cN/Tex) }\end{array}$ & $\begin{array}{c}\text { Average Elongation } \\
\text { at break (\%) }\end{array}$ \\
\hline $100 \%$ CF_0\%PVA_80T & 800 & 100 & 0 & $31.7 \pm 4.9$ & $2.7 \pm 0.4$ \\
\hline $\begin{array}{l}60 \% \text { CF_40\%PVA_80T } \\
\text { (before dissolving of PVA) }\end{array}$ & 800 & 60 & 40 & $16.7 \pm 2.3$ & $2.8 \pm 0.7$ \\
\hline $\begin{array}{l}60 \% \text { CF_40\%PVA_80T } \\
\text { (after dissolving of PVA) }\end{array}$ & 540 & 89 & 11 & $38.0 \pm 7.7$ & $1.5 \pm 0.2$ \\
\hline $\begin{array}{l}60 \% \text { CF_40\%PVA_118T } \\
\text { (before dissolving of PVA) }\end{array}$ & 800 & 60 & 40 & $14.7 \pm 2.6$ & $3.2 \pm 1.2$ \\
\hline $\begin{array}{l}60 \% \text { CF_40\%PVA_118T } \\
\text { (after dissolving of PVA) }\end{array}$ & 740 & 74 & 26 & $22.3 \pm 4.8$ & $1.5 \pm 0.3$ \\
\hline
\end{tabular}

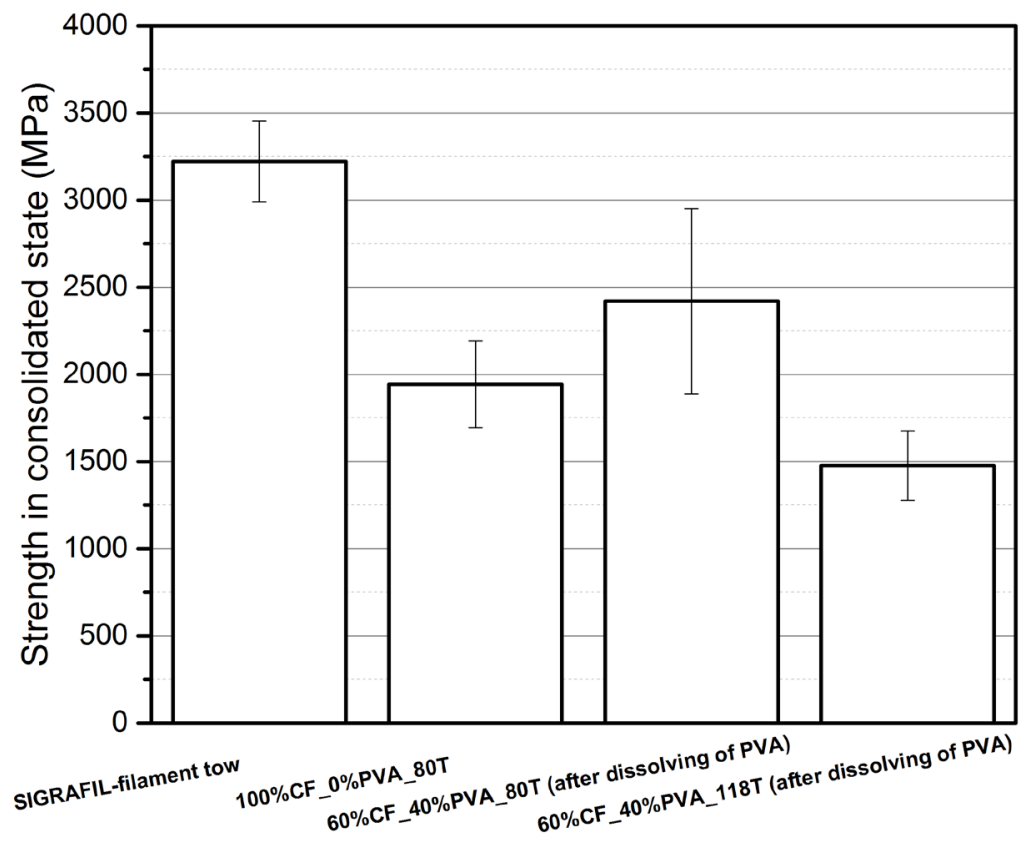

Figure 9: Comparison of strength of yarns in consolidated state 
consolidated state are illustrated in Figure 10. The results of the mechanical properties carried out on yarns/hybrid yarns in consolidated state are presented in detail in Table 6.

\section{Conclusions}

In this research work, the aspect of mixing PVA fibers with staple CF regarding the processability of $\mathrm{CF}$ in carding, drawing and spinning is investigated. Furthermore, the mechanical properties of hybrid yarns consisting of staple CF and PVA fibers, as well as yarn with $100 \%$ staple CF are investigated and compared. The investigations show that the fiber loss in carding is lower if PVA fibers are mixed with staple CF as compared to that in the case of processing of $100 \%$ staple CF due to the gentle processing of CF resulted from higher fiber to fiber cohesion. Furthermore, the slivers produced from staple CF and PVA exhibit higher fiber to fiber cohesion, as well as higher regularity. The evenness of hybrid yarns consisting of staple CF and PVA is comparatively higher than that of yarn with $100 \%$ CF. The PVA component of the hybrid yarn is subsequently dissolved using a hot water treatment.

The results of the tensile strength measurement show that the tensile strength of hybrid yarns consisting of staple CF and PVA increases significantly after hot water treatment. The remaining PVA on the hybrid yarn contributes to the increase of the tensile strength of hybrid yarns. The amount of PVA remaining on hybrid yarns depends on the level of twist of the hybrid yarn and increases with the increase of twist. The tensile test of the yarns in consolidated state reveals that the hybrid yarn produced with $80 \mathrm{~T} / \mathrm{m}$ after hot water treatment exhibits approximately $75 \%$ of the tensile strength of virgin filament tow. Therefore, it is expected that the hybrid yarns can be applied for the manufacturing of thermoset based composites for load bearing structures. However, the effect of remaining PVA in the composite on the tensile properties of composites still needs to be investigated.

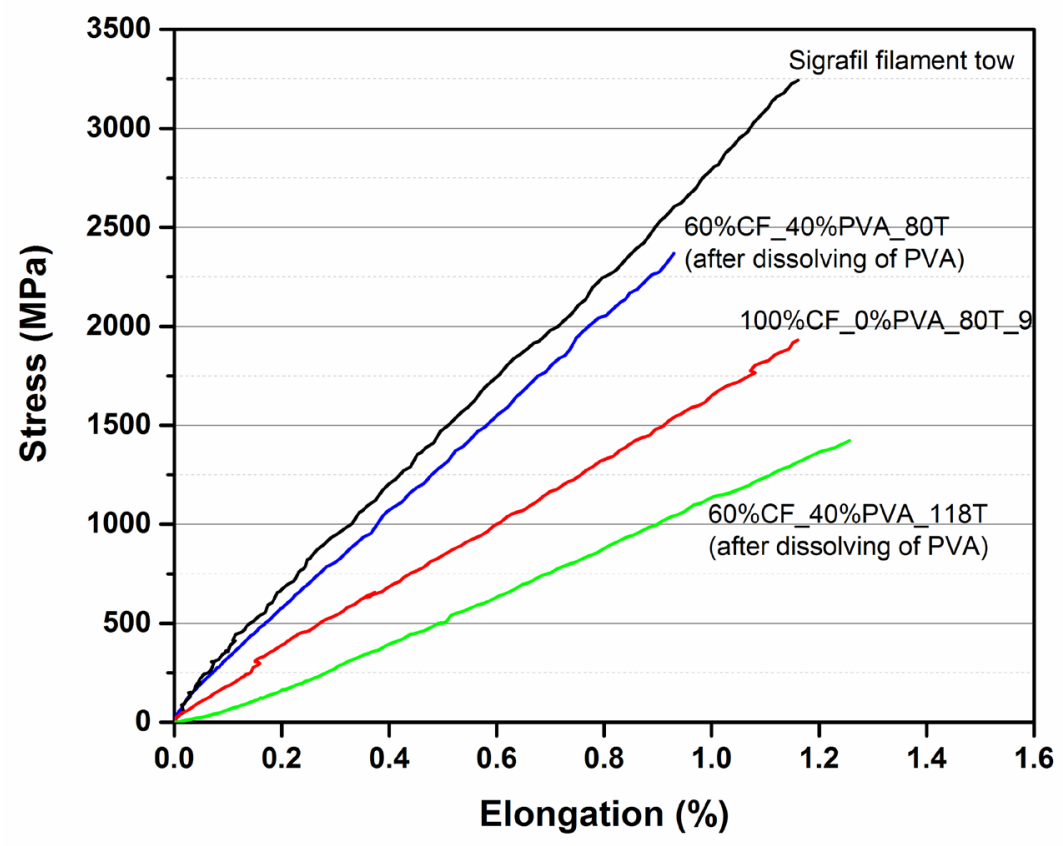

Figure 10: Typical stress strain curve of consolidated yarn specimen

Table 6: Tensile strength, E-module and elongation at break of consolidated yarn specimen

\begin{tabular}{|l|c|c|c|}
\hline Identification of composite specimen & $\begin{array}{c}\text { Tensile strength } \\
(\mathbf{M P a})\end{array}$ & $\begin{array}{c}\text { E-module } \\
(\mathbf{G P a})\end{array}$ & $\begin{array}{c}\text { Elongation at break } \\
(\%)\end{array}$ \\
\hline $\begin{array}{l}\text { SIGRAFIL-filament tow } \\
\text { 100\%CF_0\%PVA_80T } \\
\text { 60\%CF_40\%PVA_80T (after dissolving of PVA) } \\
\text { 60\%CF_40\%PVA_118T (after dissolving of PVA) }\end{array}$ & $3222.0 \pm 231.6$ & $237.0 \pm 13.2$ & $1.3 \pm 0.1$ \\
\hline 100\%CF_0\%PVA_80T & & & $1.1 \pm 0.1$ \\
\hline 60\%CF_40\%PVA_80T (after dissolving of PVA) & $1942.6 \pm 248.2$ & $170.0 \pm 40.0$ & $0.9 \pm 0.1$ \\
\hline $60 \%$ CF_40\%PVA_118T (after dissolving of PVA) & $1476.7 \pm 200.0$ & $94.4 \pm 20.0$ & $1.2 \pm 0.1$ \\
\hline
\end{tabular}




\section{Acknowledgement}

This article presents parts of results from the research program of the German Research Foundation (DFG CH 174/34-1) at the Technische Universität Dresden.

\section{References}

[1] Kraus T., Kühnel, M. (2014). Composites Market Report. Report, Carbon Composites e.V.

[2] Mazumdar, S. (2001). Composites manufacturing: materials, product, and process engineering. CRC press.

[3] Lässig, R., Eisenhut, M., Mathias. A., Schulte. R. T., Peters. F., Kühmann, T., Waldmann, T., Begemann, W.S. (2012). Series production of high-strength composites.

[4] McConnell, V.P. (May/April 2010). Launching the carbon fiber recycling industry, Reinforced Plastics.

[5] Azzam, H.A. (2007). Compressive properties of stretchbroken carbon fiber (SBCF)/ polyamide 12 commingled unidirectional composites. AUTEX Research Journal, Vol.7, No. 3.

[6] Heltra Incorporated. (1989). Hybrid yarn. WO 89/01999.

[7] Guevel, J., Francoi,s M., Bontemps, G. Carbon fiber yarn. (1989). US patent 4825635.

[8] SIGRAFIL C SBY 70. http://www.sglgroup.com/cms/ international/press-lounge/news/2013/11/11192013_p. html?_locale=en (retrieved 01 June 2016).

[9] Hengstermann, M., Raithel, N., Abdkader, A., Hasan, M.M.B., Cherif,Ch.(2015).Development of new hybrid yarn construction from recycled carbon fibers ( $r C F)$ for high performance composites. Part-l: Basic processing of hybrid CF/PA 6 yarn spinning from virgin CF staple fibers. Textile Research Journal, DOI 0040517515612363.

[10] Hengstermann, M., Abdkader, A. and Cherif, Ch. (2015). New yarn constructions from recycled carbon staple fibers and thermoplastic fibers for composite. In: Proceedings. 54. ChemiefasertagungDornbirn, Dornbirn, Austria.
[11] Hengstermann, M., Raithel, N., Abdkader, A., Cherif, Ch. (2015). Spinning of staple hybrid yarn from carbon fiber wastes for lightweight constructions. Presentation / 20. Symposium Verbundwerkstoffe und Werkstoffverbunde, Wien, Austria.

[12] Hengstermann, M., Raithel, N., Abdkader, A., Cherif, Ch. (2015). Spinning of staple hybrid yarn from carbon fiber wastes for lightweight constructions. Materials Science Forum, 825-826. DOI: 10.4028/www.scientific.net/ MSF.825-826.60, pp. 695-698.

[13] Hengstermann, M., Abdkader, A., Cherif,Ch.(2015). Production of innovative spun hybrid yarns made from recycled carbon fibers for high strength CFRP. In: Proceedings. 15th AUTEX World Textile Conference, Bucharest, Romania.

[14] Akonda, M., Lawrence, C.A., Weager, B.M. (2012). Recycled carbon fibre-reinforced polypropylene thermoplastic composites. Composites Part A: Applied Science and Manufacturing, 43 (1), 79-86.

[15] Ehrenstein, G.W. Faserverbund-Kunststoff.(2006). Carl Hanser Verlag. München. (German)

[16] http://www.kuraray.co.jp/kii/english/ (retrieved 01 June 2016).

[17] http://www.sglgroup.com/cms/_common/downloads/ products/product-groups/cf/carbon-fiber-continuous-tow/ SIGRAFIL_Carbon_Endlosfasern_d.pdf (retrieved 10 August 2016)

[18] Shayed, M. A., Hund, R. D., Cherif, C. (2012). Polysilazanebased heat-and oxidation-resistant coatings on carbon fibers. Journal of Applied Polymer Science, 124(3), 2022202.

[19] Dorigato, A., Alessandro, P. (2012). Biodegradable singlepolymer composites from polyvinyl alcohol. Colloid and Polymer Science, 290(4), 359-370. 